\title{
UNIVERSITY OF TORONTO
}

LIBRARIES

\section{CPOJ}

\section{Cänadian Prosthetics \& Orthotics Journal}

All articles are permanently available online to the public without restrictions or subscription fees. All articles are free to be used, cited, and distributed, on condition that appropriate acknowledgment is included. Authors are the copyright holders of their original contributions and grant the Canadian Prosthetics \& Orthotics Journal (CPOJ) a license to publish the article and identify itself as the original publisher. CPOJ articles are licensed under the Creative Commons Attribution 4.0 International License.

(c) $\underset{\mathrm{EY}}{(\mathbf{B}}$

CPOJ Website: https://jps.library.utoronto.ca/index.php/cpoj/index

Editorial Office: cpoj@online-publication.com

ISSN 2561-987X 


\title{
HOW INFILL PERCENTAGE AFFECTS THE ULTIMATE STRENGTH OF 3D-PRINTED TRANSTIBIAL SOCKETS DURING INITIAL CONTACT
}

\author{
Campbell $\mathrm{L}^{1}$, Lau $\mathrm{A}^{1^{*}}$, Pousett $\mathrm{B}^{2}$, Janzen $\mathrm{E}^{3}$, Raschke $S . \mathrm{U}^{3}$ \\ ${ }^{1}$ Prosthetics and Orthotics, School of Health Sciences, British Columbia Institute of Technology (BCIT), Burnaby, British Columbia, \\ Canada. \\ ${ }^{2}$ Barber Prosthetics Clinic, Vancouver, British Columbia, Canada. \\ ${ }^{3}$ MAKE + Applied Research, Centre for Applied Research \& Innovation (CARI), Burnaby, British Columbia, Canada.
}

\section{ABSTRACT}

BACKGROUND: 3D printing is becoming more popular across many industries. The first step to safely introducing $3 D$ printed sockets in to prosthetics is to conduct strength testing on these sockets.

PURPOSE: This study tests how changing the infill percentage (the percentage of material between the internal and external socket wall) affects the strength of 3D-printed transtibial sockets.

METHODS: A Fused Deposition Modelling (FDM) printer was used to print a total of nine transtibial (TT) sockets (three sockets at $30 \%$ infill, three sockets at $40 \%$ infill, and three sockets at $50 \%$ ) using polylactic acid (PLA). A strengthtesting apparatus measured, in Newtons $(\mathrm{N})$, the maximum load the 3D-printed transtibial sockets could withstand at initial contact of the gait cycle.

RESULTS: Based on the specific criteria outlined in this research project, all nine sockets exceeded the $4480 \mathrm{~N}$ threshold set by ISO Standard 10328. Eight out of nine sockets failed at approximately double the force required with one socket (socket \#2) failing at $5360 \mathrm{~N}$. Seven out of nine sockets failed at the medial popliteal region and two out of nine sockets failed at lateral mid socket region. Differences in infill percentage from 30\%, 40\%, 50\% did not appear to influence strength of sockets.

CONCLUSION: Strength of 3D-printed TT sockets needs rigorous testing to be deemed safe for patient use. More definitive research and a higher number of samples are required to investigate how a larger range of infill percentage can affect strength. Until all the requirements of ISO Standard 10328 are satisfied, the safety of using 3D-printed TT sockets in clinical practice are uncertain.

\section{ARTICLE INFO}

Received: August 21, 2018 Accepted: September 21, 2018 Published: September 28, 2018

\section{CITATION}

Campbell L, Lau A, Pousett B, Janzen E, Raschke S.U. How infill percentage affects the ultimate strength of 3D-printed transtibial sockets during initial contact. Canadian Prosthetics \& Orthotics Journal, Volume 1, Issue 2, No 2, 2018. https://doi.org/10.33137/cpoj.v1i 2.30843

\section{KEYWORDS}

Prosthetic socket, Transtibial, 3D Printing, Infill percentage, Strength testing, Fused deposition modeling, Polylactic acid.

\section{ABBREVIATIONS}

FDM: Fused Deposition Modeling Fmax: Maximum force Fset: Settling test force Fsp: Static proof test force PLA: Polylactic Acid

TIJ: Thermal Inkjet Printing SLS: Selective Laser Sintering

\section{*CORRESPONDING AUTHOR}

Adriel Lau, Prosthetics and Orthotics, School of Health Sciences,

British Columbia Institute of Technology (BCIT), 3700 Willingdon Avenue, Burnaby, British Columbia, Canada.

Email: lau.adriel@gmail.com

DOI: https://doi.org/10.33137/cpoj.v1i2.30843 


\section{INTRODUCTION}

3D printing has been around for 30 years, but recently it has been making significant strides in the field of prosthetics. Some prosthetics and orthotics clinics have begun to use the technology in their practice due to the decrease in cost of 3D printers as well as the development of 3D scanning technology to efficiently capture the shape of a patient's limb. ${ }^{1}$ 3D printing allows for increased cost efficiency and enhanced productivity. ${ }^{2}$ Because of this, 3D-printed prosthetic sockets are becoming an alternative option to traditional methods. However, 3D-printed sockets used for weight bearing should be tested rigorously to ensure patient safety.

Ventola (2014) states that the three most common types of 3D printers used in medical applications are selective laser sintering (SLS), thermal inkjet (TIJ) printing, and fused deposition modeling (FDM). ${ }^{2}$ During SLS printing, a laser draws the shape of the object in powder which fuses it together. TIJ printing "uses thermal, electromagnetic, or piezoelectric technology to deposit tiny droplets of 'ink' onto a substrate according to digital instructions". 2 FDM printers lay down layers of heated beads of plastic and these build the object layer by layer. FDM printers are less expensive and more common than the SLS type printers. ${ }^{2}$ For this reason, they are likely the type of printer that prosthetics and orthotics clinics will have in their clinics. ${ }^{2}$

The technology of $3 D$ printing is advancing, but many of these designers are becoming more involved with the idea of creating prostheses when they have little to no concept of the intricacies of creating a prosthetic device nor the treatment planning involved. For example, Chhaya et al (2015) discuss several online groups that provide opensource files available for printing upper extremity devices. ${ }^{3}$ As both prosthetists and healthcare professionals, we believe there is a need to become more involved in the process of designing and testing safe 3D-printed sockets.

Before additive manufacturing technology can be fully implemented in the prosthetics and orthotics field, there are barriers that must be addressed and resolved. ${ }^{4}$

\section{Strength Testing}

To ensure that weight bearing 3D-printed sockets are safe for patient use, they should adhere to the strength standards for lower extremity prostheses. ISO Standard 10328 is the international standard for the structural testing of lower limb prostheses and it outlines test methods. ${ }^{5}$

ISO 10328 outlines the procedures for testing lower limb prostheses both statically and cyclically. ${ }^{5}$ The static test is a one-time, single-event test to determine the performance of a structure under a specific load. The cyclic test consists of a specific load applied to a structure multiple times or for many cycles, simulating conditions of normal walking. ${ }^{5}$

Lower extremity prostheses must be tested to satisfy ISO 10328 standards loading condition I and II. Condition I loading "the instant of maximum loading occurring early in the stance phase of walking". ${ }^{5}$ Condition II loading "the instant of maximum loading occurring late in the stance phase of walking". ${ }^{5}$

There are three loading levels that can be tested in ISO 10328. P5 is the loading condition that based on data from amputees with body masses are above and below $100 \mathrm{~kg}$. P4 condition is for an amputee whose body mass is less than $80 \mathrm{~kg}$, and finally $\mathrm{P} 3$ condition is less than $60 \mathrm{~kg} \cdot{ }^{5}$

A literature search for the strength testing of $3 D$ printed lower extremity prostheses did not produce any results. Other studies have used ISO standards to test the strength of non-3D printed lower extremity prostheses.

Gerschutz et al., (2012) conducted a study examining static failure loads on traditional sockets. ${ }^{6}$ In their study, mechanical testing on three different types of sockets was performed (thermoplastic check, copolymer and definitive laminated) for a total of $\mathrm{n}=98$ sockets. They were assessed for passing brittle failure $=4,426 \mathrm{~N}$ and ductile failure $=3,421 \mathrm{~N}$. This quantitative study evaluated socket strength to provide an understanding of the materials used for prosthetic sockets.

Goh et al., (2002) tested complete prostheses using both cyclic and static procedures. ${ }^{7}$ The researchers conducted the static test procedure by subjecting the prosthesis to sinusoidal loads ranging from $50 \mathrm{~N}$ to Fmax (Fmax=1330N for condition I and Fmax $=1200 \mathrm{~N}$ for condition II). The force was maintained for 30 s and if permanent deformation of $15 \mathrm{~mm}$ or the prosthesis failed, the prosthesis was not found to be adhered to ISO 10328 standards. ${ }^{7}$ If the prosthesis did not fail, it proceeded to the failure 
test. The article found that the polypropylene sockets passed the static load test with only minimal deformation and passed cyclic test of 250000 cycles with no failure.

\section{Infill percentage}

3D printing can change different parameters such as infill percentage. Infill percentage is the density of the plastic between the inner and outer walls of a printed object. ${ }^{8}$ Increasing the infill percentage on a 3Dprinted transtibial (TT) socket made from polylactic acid (PLA) should influence the ultimate strength of the socket. Johansson (2016) states that low infill is weaker and more prone to cracking. ${ }^{8}$ Whereas, printing at $100 \%$ can increase printing time and cost and may not necessarily increase strength. ${ }^{8}$ It is valuable for the prosthetics and orthotics profession to determine at what infill percentage has adequate strength, but at the same time, does not take an excessive amount of time to print.

The purpose of this study was to test how changing the infill percentage affects the strength of a 3Dprinted TT socket during initial contact. We hypothesized that greater infill percentage could correspond to a greater socket strength. This project collaborated with a research facility to investigate this concept and hope to be a part of a much bigger picture in progressing $3 \mathrm{D}$ printing as a tool to help the field of prosthetics.

\section{METHODS}

\section{Settings}

This experimental study printed nine identical Total Surface Bearing (TSB) transtibial sockets. To maintain consistency, the sockets were all printed from the same data file supplied by the company Additive O\&P in Charlotte, N.C. and Barber Prosthetics Clinic in Vancouver, B.C.

The sockets were printed from a FDM printer located at Barber Prosthetics Clinic. The FDM printer uses a nozzle head that extrudes melted plastic layer by layer to create a three-dimensional structure. ${ }^{2}$ It took an average of 8-9 hours to print each socket. The sockets were all printed using a white PLA filament and were all reinforced with scotch cost. At the distal end, each socket was attached to a 5R1 wood block which served as the attachment point for the pylon in the strength testing apparatus. A Registered Prosthetic Technician oversaw the production and finished each 3D-printed transtibial socket as can be seen in Figure 1.

Considering the various $3 \mathrm{D}$ printer parameters that were available to be evaluated, the authors chose to research infill percentage as a parameter because this variable was hypothesized to have a direct relationship with strength and print time. Three different infill percentages were chosen that would best represent realistic categories that clinicians may decide to print: Three sockets were printed at $30 \%$ infill, three sockets at $40 \%$ infill, and three sockets at $50 \%$ infill. Table 1 describes the properties of each socket.

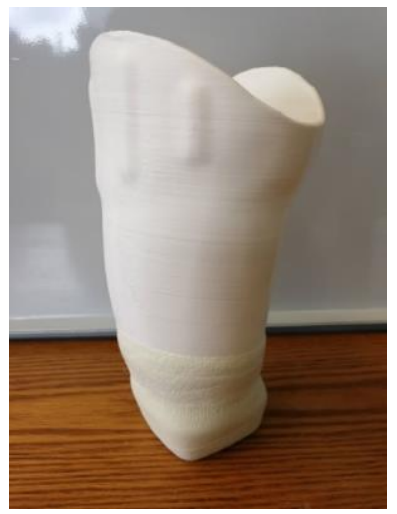

Figure 1. 3D-Printed TT Socket

Table 1. Characteristics of the 3D-Printed Sockets

\begin{tabular}{c|c|c|c|c}
\hline Socket \# & Infill \% & $\begin{array}{c}\text { Length } \\
(\mathrm{cm})\end{array}$ & $\begin{array}{c}\text { Weight - } \\
\text { Unfinished (gr) }\end{array}$ & $\begin{array}{c}\text { Finished } \\
(\mathrm{gr})\end{array}$ \\
\hline 1 & 30 & 20.8 & 291 & 531 \\
\hline 2 & 30 & 20.8 & 304 & 543 \\
\hline 3 & 30 & 20.8 & 304 & 543 \\
\hline 4 & 40 & 20.8 & 297 & 527 \\
\hline 5 & 40 & 20.8 & 296 & 538 \\
\hline 6 & 40 & 21 & 302 & 543 \\
\hline 7 & 50 & 20.8 & 325 & 564 \\
\hline 8 & 50 & 20.9 & 324 & 574 \\
\hline 9 & 50 & 20.5 & 326 & 575 \\
\hline
\end{tabular}

\section{Componentry}

This project worked closely with the Centre for Applied Research Institute (CARI) in Burnaby, BC to fabricate components that held the 3D-printed socket in the proper orientation for strength testing as can be seen in Figure 2. The components included an upper plate and bottom plate made from one-inch thick steel. The superior part of the upper plate and the inferior part of the lower plate contain a concave surface that articulated with two hitch balls which was attached to the strength testing apparatus. Firstly, this ensured a pure vertical force was generated as accurately and consistently as possible. Secondly, 
this allowed the plates to be oriented in the correct alignment for this project. This experiment focused on initial contact of the gait cycle as ISO 10328 states that during this phase, the socket experiences the largest loading force. ${ }^{5}$ Lastly, the plates can be placed in different orientations to test for multiple weight classes which are specified as P3, P4, and P5. Our study focused on P5 condition which is the loading condition that based on data from amputees with body masses are above and below $100 \mathrm{~kg}$.
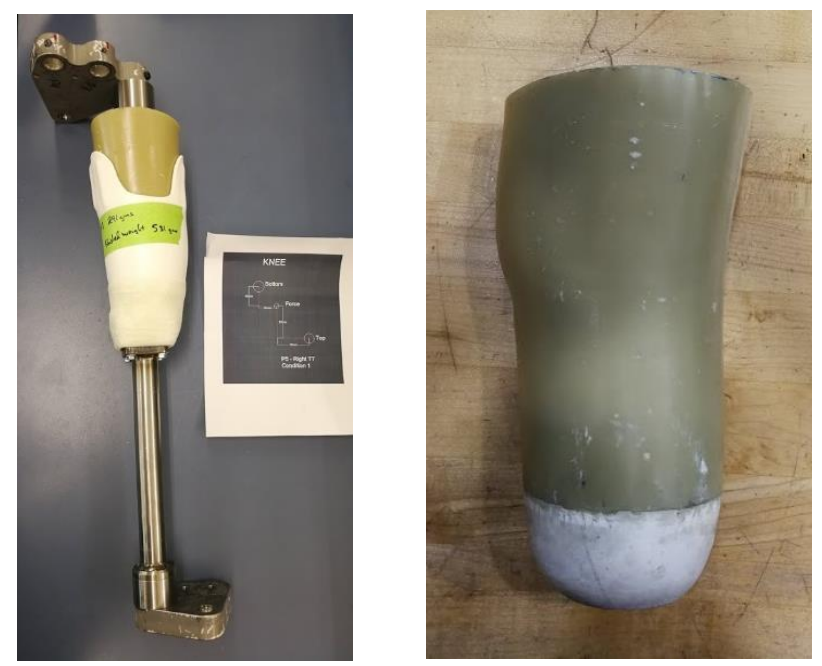

Figure 2 (Left). Componentry Attached to 3D-Printed Socket.

Figure 3 (Right). High-Density Urethane Residual Limb Model.

To simulate a residual limb, a high-density urethane mold was fabricated as seen in Figure 3. The model was created from a mold of the socket shape which created an intimate fit thus simulating an appropriate fit with the TSB socket. The urethane mold and the upper plate were connected by a $5 / 8$ bolt which allowed the load generated by the strength testing apparatus to be distributed throughout the entire socket. The bottom plate articulated with the socket through a solid piece of welded steel that acted as the pylon.

Since existing prosthetic componentry such as the pylon, tube clamp adaptors and pyramids have already been tested to ISO standards, attempting to eliminate them from the equation is most logical to isolate the strength of the socket. Therefore, a $311 \mathrm{~mm}$ solid piece of steel was fabricated to satisfy these requirements. This piece of steel also contained a welded pyramid adaptor which allowed it to be attached to the wood block.

\section{Strength Testing Apparatus}

Strength testing was performed on a Tinius Olsen Universal testing machine as seen in Figure 4 (Left) and focused solely on static testing. Cyclic testing requires 3 million cycles, ${ }^{5}$ which would take approximately one year of continuous testing. Due to time and cost constraints, only ultimate strength testing was completed.

The sockets were tested for ultimate strength. Ultimate strength is defined as static load representing a gross single event, which can be sustained by the prosthetic device/structure but which could render it unusable. ${ }^{5}$ The static load will be applied for testing condition I which is described as evaluating the instant of maximum loading during early stance phase or initial contact of the gait cycle. Each socket was tested to ISO standards where the force measurement data was gathered in Newtons (N). $4480 \mathrm{~N}$ was the force required in order to pass the standards outlined in ISO $10338 .{ }^{5}$ Furthermore, if the sockets surpassed the threshold, they were subjected to further testing and compressed to failure to determine how much force is required before breaking the socket.

The procedure for principal static ultimate strength test as per ISO standard 10328 is as follows:

A force was steadily increased at a constant rate between $100 \mathrm{~N} / \mathrm{s}$ and $250 \mathrm{~N} / \mathrm{s}$ to $2240 \mathrm{~N}$ and held for 10-30 seconds (Values are recorded). The force was removed and the socket rested at zero load for one minute. The force was again steadily increased at a constant rate between $100 \mathrm{~N} / \mathrm{s}$ and $250 \mathrm{~N} / \mathrm{s}$ to 4480 $\mathrm{N}$ and held for 30 seconds (Values are recorded). If the socket had not failed, a compression force continued and the load was increased until failure was reached. Figure 4 (Right) provides a visual representation of the 3D-printed socket attached to all the componentry.

\section{RESULTS}

\section{Force at Socket Failure}

The results of the strength testing procedure were recorded in Table 2. These recordings include the settling test force (Fset), the amount of time each socket spent at Fset, the amount of time the socket spent with no force between Fset and the static proof test (Fsp), the actual force at Fsp, the time the socket spent at Fsp, the force that the socket ultimately 
failed at, and whether the socket passed the minimum force dictated in the ISO Standard 10328.
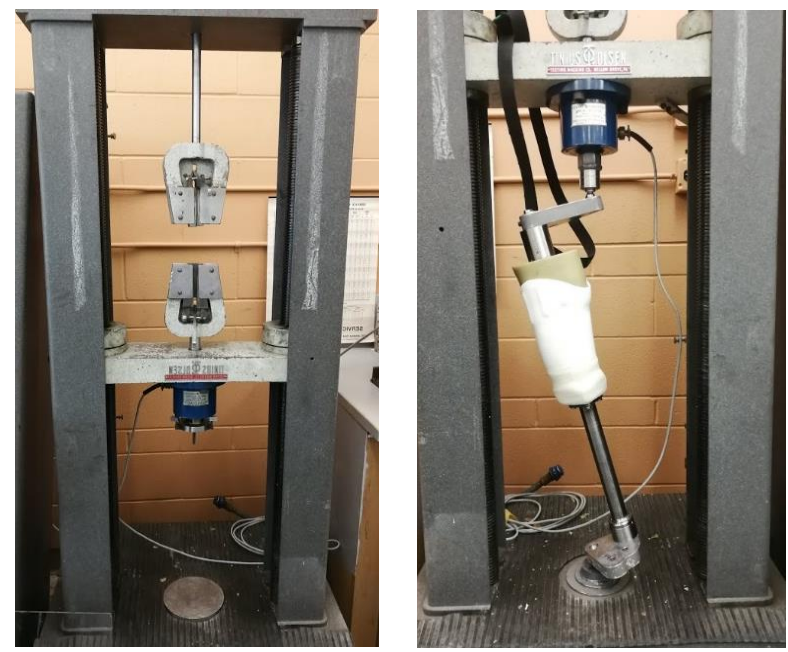

Figure 4. Left: Tinius Olsen Universal Testing Apparatus; Right: Completed set-up.

Table 2. Results of Loading Procedure (Note: Fset = Settling test force, Fsp = Static proof test force).

\begin{tabular}{|c|c|c|c|c|c|c|c|c|}
\hline 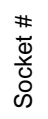 & 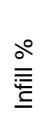 & $\begin{array}{l}\bar{z} \\
\bar{\Phi} \\
\stackrel{\omega}{\omega}\end{array}$ & 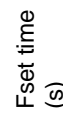 & 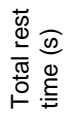 & $\begin{array}{l}\widehat{z} \\
\text { o } \\
\text { ए) }\end{array}$ & 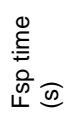 & 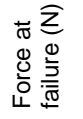 & 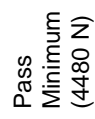 \\
\hline 1 & 30 & 1079 & 15 & 60 & 2630 & 30 & 11854 & $Y$ \\
\hline 2 & 30 & 1074 & 10 & 60 & 2438 & 30 & 5360 & $Y$ \\
\hline 3 & 30 & 1070 & 10 & 60 & 2303 & 30 & 12841 & $Y$ \\
\hline 4 & 40 & 1114 & 10 & 60 & 2314 & 30 & 9009 & $Y$ \\
\hline 5 & 40 & 1147 & 10 & 60 & 2425 & 30 & 10345 & $Y$ \\
\hline 6 & 40 & 1216 & 10 & 60 & 2266 & 30 & 11383 & $Y$ \\
\hline 7 & 50 & 1252 & 10 & 60 & 3457 & 30 & 11965 & $Y$ \\
\hline 8 & 50 & 1115 & 10 & 60 & 2371 & 30 & 12243 & $Y$ \\
\hline 9 & 50 & 1177 & 10 & 60 & 2491 & 30 & 9847 & $Y$ \\
\hline
\end{tabular}

Both the Fset and Fsp forces listed in table 2 are different for each socket due to the setting capabilities of the strength testing apparatus. The apparatus was controlled via a dial, so a specific set point for both Fset and Fsp was not possible. The accuracy of these points depended solely on the operator of the strength testing apparatus pausing the loading at a force close to the intended forces (laid out in the methods) of Fset $=1024 \mathrm{~N}$ and Fsp= $2240 \mathrm{~N}$.

Figure 5 shows the force at failure of all 3D-printed sockets. The black threshold line indicates the minimum threshold $(4480 \mathrm{~N})$ that the ISO standards dictates for condition I, weight class P5. As shown in Figure 6, all the sockets surpassed the threshold and all sockets (with the exception of socket number 2) failed at approximately double the force required by ISO standards

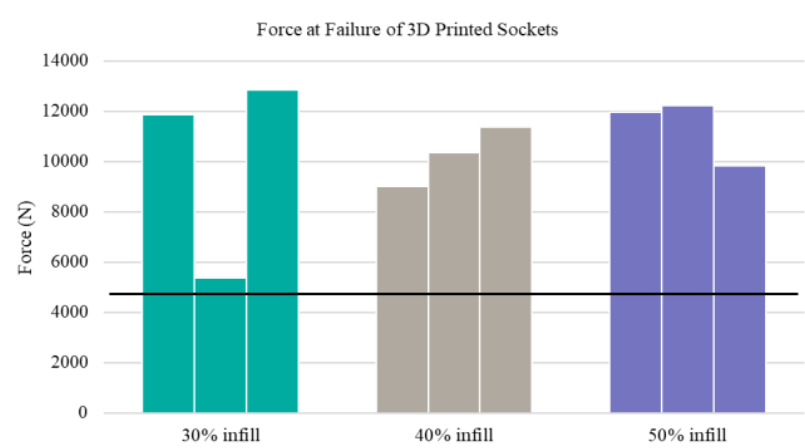

Figure 5. All 3D printed transtibial sockets failed above the ISO standard for this condition.

\section{Visual Analysis of Socket Failure}

Table 3 shows the area that the sockets failed at as well as the type of socket failure. Most sockets failed in the medial popliteal region (seven of nine sockets) and two of the nine sockets failed in the lateral mid socket region. An example of a medial popliteal area socket failure is shown in Figure 6 (left). Figure 6 (right) shows an example of a socket failing in the lateral mid socket.

Table 3. Area of Socket Failure and Failure Type

\begin{tabular}{c|c|c|c} 
Socket \# & Infill \% & Failure point & Failure Type \\
\hline 1 & 30 & medial popliteal & crack \\
\hline 2 & 30 & lateral mid socket & crack \\
\hline 3 & 30 & medial popliteal & complete \\
\hline 4 & 40 & medial popliteal & crack \\
\hline 5 & 40 & lateral mid socket & crack \\
\hline 6 & 40 & medial popliteal & crack \\
\hline 7 & 50 & medial popliteal & complete \\
\hline 8 & 50 & medial popliteal & crack \\
\hline 9 & 50 & medial popliteal & crack
\end{tabular}
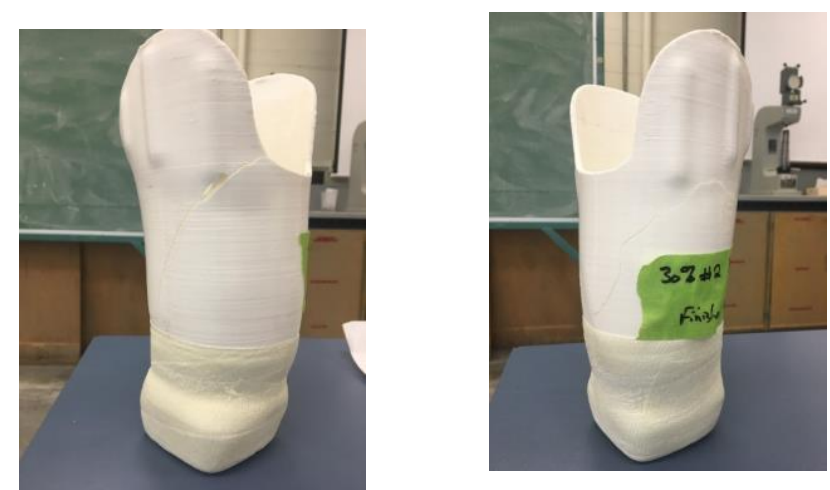

Figure 6. Left: Socket broken in the medial popliteal area; Right: Socket broken in the middle lateral area. 
Table 3 shows that the primary method for socket failure was a crack in the socket (seven of the nine sockets). A crack failure type was defined as a failure in which the proximal and distal aspects of the socket remained attached. Figure 6 shows examples of a crack failure. Figure 7 shows an example of a complete failure of the socket. In this case, the proximal and distal aspects of the socket are completely separated from each other. Two of the nine sockets failed in this manner.

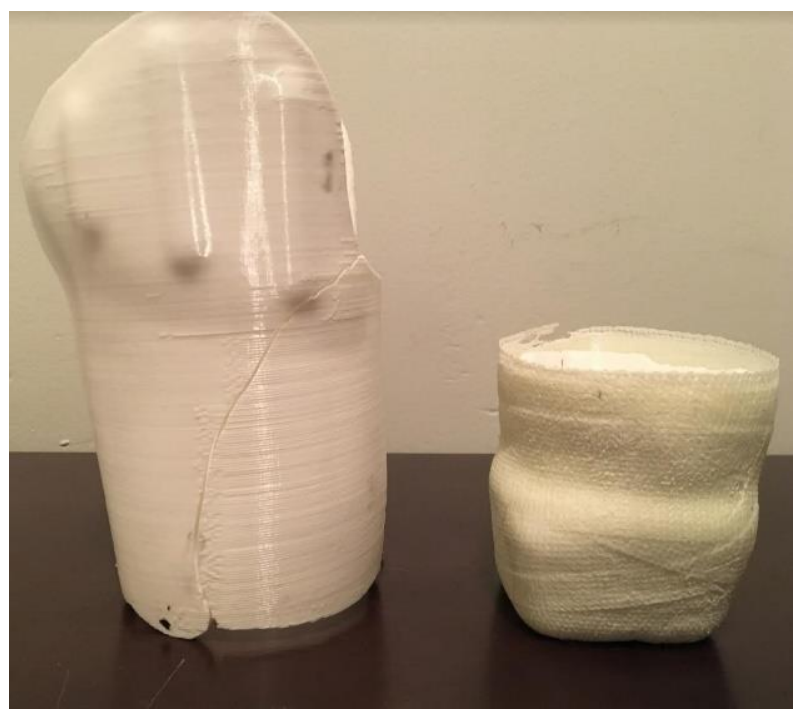

Figure 7: Complete Socket Failure

\section{DISCUSSION}

The purpose of this research project was to evaluate how changing infill percentage would affect the ultimate strength of a 3D-printed TT socket. To safely use these sockets in either a diagnostic or a definitive phase, the efficacy and effectiveness of these devices must be clearly shown. ${ }^{9}$ 3D-printed sockets should satisfy the same requirements as lower limb conventional prostheses as stated in ISO Standards 10328. Based on the results, the sockets in this study exceeded the threshold of $4480 \mathrm{~N}$. However, it is important to note that the sockets were evaluated with a specific set of criteria. The conditions of static testing, initial contact (condition I) and P5 weight class were implemented and only under these conditions did all of the sockets exceed the threshold. To fully deem a 3D-printed socket safe for patient use, the other criteria explained in ISO standard 10328 will also need to be satisfied. For example, cyclic testing needs to be performed where the socket is continuously compressed through a low load, long duration setting. This will better simulate a socket when completing activities of daily living.
Furthermore, it has been seen in clinical practice that sockets often fail during terminal stance in the gait cycle. Testing in this specific phase of the gait cycle in addition to initial contact, will provide a more encompassing picture as an individual ambulates. Testing each of these conditions in all the weight classes (P3, P4 and P5) will also need to be completed to fully satisfy safety requirements.

The infill percentage did not appear to influence the ultimate strength of the sockets. It was hypothesized that a greater infill percentage would require a greater force for a socket to fail. However, the results show that all the sockets failed at approximately twice the required force (except for socket \#2). Perhaps the range of infill percentage was not large enough to see a difference in ultimate strength. In the future, choosing a larger gap in infill percentage may show difference in the force values required for a socket to fail. Another possible explanation for this result is that the other parameters used for the sockets were structurally weaker so the infill percentage was not the factor that influenced failure.

The failure points occurred in different areas of the socket during the experiment; however, it was observed that all the failure points occurred above the reinforced scotch cast. With a diagnostic socket, it is more advantageous that reinforcement methods be done to create additional strength and stability. With the breaks occurring above this area, this suggests that the reinforced area is successful in delegating the force to an area that is less strong. Knowing this, removing the reinforced scotch cast in might have shown different results when evaluating for ultimate strength. As mentioned earlier, the sockets broke in different areas with seven of the nine sockets failing on the medial popliteal area and two of the nine sockets failing at the middle, lateral area of socket. Two of the sockets also experienced complete failure where the socket was broken into two separate pieces. There was no predictability and no trend was seen as to how a socket broke compared to its failure values.

\section{Limitations}

The limitations of this study included the small sample size of sockets. Due to cost and time constraints, only nine sockets were printed as this was a logical way to compare sockets printed at different infill percentages. Because of the small sample size, we were unable to comment on any significant differences and can only comment on 
general trends. Future studies should focus on printing a larger number of sockets encompassing a wider variety of infill percentages which could enable the generation of a statistical analysis.

In general, more testing is needed to be done on the strength of 3D-printed sockets. The settings of 3D printing can be optimized and customized to the user or practitioner's preferences. As a result, evaluating how individual parameters affect the strength of a socket would be beneficial. This study focused on infill percentage as an attempt to isolate its effects and how changing this setting would ultimately affect its strength.

No articles were found regarding the evaluation of the ultimate strength of a 3D-printed TT socket. 3D printing is a relatively new technology in the field; therefore, there is not enough data currently to perform meta-analysis. There are also no randomized control trials to investigate the efficacy of these sockets which created a challenge in narrowing down the testing procedures and methodology process. With 3D printing technology advancing at an impressive rate, future directions should consider investigating how different printers, different materials and how different methods of printing can affect the strength of a socket.

\section{CONCLUSION}

3D printing technology is currently being used in many different industries. The field of prosthetics and orthotics needs to embrace this technology and demonstrate how it can be successfully used in clinical practice. A logical first step is testing the strength of 3D-printed prosthetic sockets to determine if it is safe for patient use. This research project demonstrated that the amount of force required for a socket to fail exceeded the $4480 \mathrm{~N}$ threshold set by ISO Standard 10328. Furthermore, infill percentages ranging from $30 \%$ to $50 \%$ did not seem to affect the ultimate strength of the sockets. However, it should be noted that the sockets were tested to specific and limited criteria (static testing, initial contact and P5 weight class). This project is a stepping stone to much more extensive research and as such, further work is recommended to investigate how different parameters can influence the strength of socket. Moreover, additional conditions outlined by the ISO standards need to also be satisfied to determine if 3D-printed prosthetic sockets are safe and suitable for patients.

\section{ACKNOWLEDGEMENT}

The authors would like to thank Dave Moe (CP), Daryl Murphy (RTP), and Malena Rapaport (CP) from Barber Prosthetics Clinic. The authors would also like to thank Additive O\&P, the Centre for Applied Research Institute, Dr. Nathan Devos and Caroline Soo for their guidance and support throughout this project.

\section{DECLARATION OF CONFLICTING INTERESTS}

Barber Prosthetics Clinic donated the use of their 3D printer and the PLA used to print the sockets. The researchers did not receive any financial compensation for this project.

\section{ETHICAL APPROVAL}

Not required

\section{AUTHOR CONTRIBUTION}

- Leah Campbell: Conceptualization, formal analysis, investigation, methodology, visualization, writing original, review \& editing.

- Adriel Lau: Conceptualization, formal analysis, investigation, methodology, visualization, writing original, review \& editing.

- Brittany Pousett: Conceptualization, investigation, visualization, supervision, review \& editing.

- Ernie Janzen: Investigation, visualization, methodology, review \& editing.

- Silvia Ursula Raschke: Conceptualization, visualization, review \& editing.

\section{REFERENCES}

1) Daly A. Socio-Legal Aspects of the 3D Printing Revolution: Palgrave Macmillan UK. 2016, DOI: 10.1057/978-1-137-51556-8.

2) Ventola C.L. Medical applications for 3D printing: Current and projected uses. P \& T: A Peer-Reviewed Journal for Formulary Management. 2014; 39(10), 704711.

3) Chhaya M.P, Poh P.S, Balmayor E.R, Griensven M, Schantz J.T, Hutmacher D.W. Additive manufacturing in biomedical sciences and the need for definitions and norms. Expert Review of Medical Devices. 2015; 12(5), 537-543. DOI:10.1586/17434440.2015.1059274 
4) Chen R.K, Jin Y, Wensman J, Shih A. Additive manufacturing of custom orthoses and prostheses-A review. Additive Manufacturing. 2016;12,77-89. Doi.org/10.1016/j.addma.2016.04.002.

5) International Organization for Standardization. Prosthetics-Structural testing of lower limb prosthesesRequirements and test methods (ISO 10328), 2006.

6) Gerschutz M.J, Haynes M.L, Nixon D, Colvin J.M. Strength evaluation of prosthetic check sockets, copolymer sockets, and definitive laminated sockets. Journal of Rehabilitation Research and Development. 2012; 49(3), 405-426. http://dx.doi.org/10.1682/JRRD.2011.05.0091

7) Goh J.C, Lee P.V, Ng P. Structural integrity of polypropylene prosthetic sockets manufactured using the polymer deposition technique. Proceedings of the Institution of Mechanical Engineers, Part $\mathrm{H}$ : Journal of Engineering in Medicine. 2002; 216(6), 359-368. https://doi.org/10.1243/095441102321032157

8) Johansson F. Optimizing fused filament fabrication 3D printing for durability: Tensile properties and layer bonding (Dissertation). 2016; Retrieved from: http://urn.kb.se/resolve?urn=urn:nbn:se:bth-12355

9) Diment L.E, Thompson M.S, Bergmann J.H.M. Threedimensional printed upper-limb prostheses lack randomised controlled trials: A systematic review. Prosthetics and Orthotics International. 2018; 42(1), 7-13. https://doi.org/10.1177/0309364617704803. 